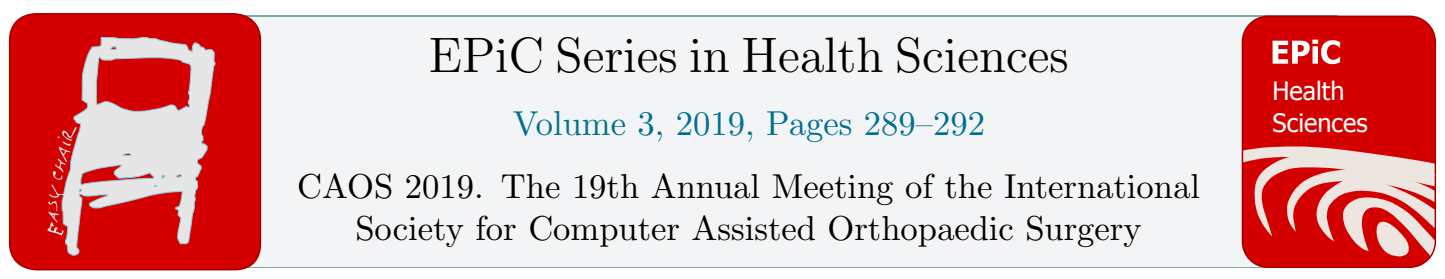

\title{
3D pose estimation with depth camera for markerless Computer Assisted Orthopaedic Surgery
}

\author{
Jérôme Ogor ${ }^{1}$, Guillaume Dardenne ${ }^{1}$, Salahhedine Sta $^{2}$, Julien Bert ${ }^{1}$, Hoël \\ Letissier $^{1}$, Eric Stindel ${ }^{1}$ and Chafiaa Hamitouche ${ }^{2}$ \\ ${ }^{1}$ LaTIM UMR1101 INSERM \\ ${ }^{2}$ Institut Mines-Télécom Atlantique Bretagne-Pays de la Loire \\ jerome.ogor@inserm.fr
}

\begin{abstract}
Surgical navigation systems have been used in orthopaedics for many years. These solutions however often mean additional time and complexity because, essentially, of the markers. We want to introduce a new solution based on depth camera which could be used intraoperatively to estimate the $3 \mathrm{D}$ pose of surgical instruments without specific markers. The goal of this paper is to assess, on synthetic data, an algorithm called Clustered Viewpoint Feature Histogram (CVFH) to estimate the pose of an orthopaedic cut guide used during knee surgeries. A specific simulator has been developed for this study which allows the simulation of a point cloud associated to the cut guide. The Average Distance Distinguishable (ADD) metric has been measured 1000 times according to several cut guide orientations and several noise levels. The success rate has also been analyzed. It is commonly considered that the pose is correctly estimated if ADD is less than $10 \%$ of the largest dimension of the object. The ADD metric and the success rate vary from $2.12 \pm 4.46 \mathrm{~mm}$ to $2.82 \pm 5.73 \mathrm{~mm}$ and from $96.0 \%$ to $92.4 \%$ for respectively a low $(0 \mathrm{~mm})$ and a high noise $(10 \mathrm{~mm})$. The results are very promising. However, more parameters have to be assessed. Similarly, the accuracy and reliability of such method have to be evaluated in a real clinical environment.
\end{abstract}

\section{Introduction}

For several years, surgical navigation systems have been used in the operating room in order to assist surgeons during surgery. These systems are mainly composed of a three-dimensional (3D) localizer which can retrieve the positions and orientations of specific markers attached either on bones or instruments. Based on these information, relevant clinical parameters can be provided to the surgeon in order to assist him during the intervention. However, because of these markers, these solutions lead to additional time, cost and complexity during the intervention. Furthermore, [1] highlights disappointing postoperative results due to the bad positioning of these instruments during the operation. Therefore we want to introduce a new solution based on a depth camera which could be 
used intraoperatively to estimate the 3D pose of surgical instruments without specific markers. Several algorithms can be used to estimate the pose of objects in a depth scene. These algorithms can be classified into two categories: local descriptors which compute geometric features on each individual point of the object and global descriptors which encode the entire object geometry. Unlike [2] which uses a local descriptor, we want to assess here, on synthetic data, a more recent global descriptor algorithm: the Clustered Viewpoint Feature Histogram (CVFH) [3] to estimate the pose of an orthopaedic cut guide used during knee surgeries.

\section{Methods}

The overall pipeline of a global descriptor is divided into two parts:

- The training phase which allows the creation of a database. This database is built by virtually computing the CVFH feature of the object under different synthetic views obtained thanks to a CAD model of the object.

- The testing phase which aims to estimate the pose in a 3D scene. In this phase, the CVFH descriptor is computed and the nearest neighbor view from the database is estimated according to the Chi-squared distance between CVFH histograms. The pose found from the database is then refined by the Iterative Closest Point (ICP) algorithm.

A specific simulator has been developed for this study. This simulator can generate a point cloud associated to an orthopaedic cut guide placed at $50 \mathrm{~cm}$ from a virtual depth camera. The resolution of this virtual camera was set to $640 \times 480$. The orientation of the cut guide with respect to the camera as well as the level of noise associated to the data provided by the camera can be modified. The noise is simulated by a Gaussian noise applied on each point of the generated point cloud. The $\varepsilon$ evaluation metric (ADD), widely used in robotics, was computed to assess the accuracy of the CVFH method and is defined according to the equation below:

Where:

$$
\varepsilon=\frac{1}{m} \sum_{x \in M} \quad(R x+T)-(R x+\mathcal{P}) \quad 2
$$

- $\mathrm{M}$ represents the object point cloud,

- $\mathrm{m}$ is the number of points in $\mathrm{M}$,

- $T$ and Rrepresent the estimated poses respectively in translation and rotation,

- $T$ and $R$ are the ground truths.

The $\varepsilon$ metric was computed 1000 times under several orientations and according to several noise levels: $0,2,4,6,8$ and $10 \mathrm{~mm}$. The success rate of the pose estimation was also analyzed. In this criterion, a pose is considered to be a success if the $\varepsilon$ metric is lower than a threshold defined as $\mathrm{k} \times \mathrm{d}$, where $\mathrm{k}$ and $\mathrm{d}$ represent respectively an adjustable parameter between 0 and 0.1 , and the maximum distance between two points of the object.

\section{Results}

The mean $\varepsilon$ metric varies from $2.12 \mathrm{~mm} \pm 4.46 \mathrm{~mm}$ to $2.82 \mathrm{~mm} \pm 5.73 \mathrm{~mm}$ for respectively a noise level of $0 \mathrm{~mm}$ and $10 \mathrm{~mm}$. Relevant statistics from all the levels of noise tests are also provided in the Table 1. The pose estimation success rate according to $\mathrm{k}$ is shown in the Figure 1. It is usually considered in the literature [4] that the pose is correctly estimated if $\varepsilon$ is less than the threshold defined with $\mathrm{k}=10 \%$. We can therefore observe from this figure that the success rate was $96.0 \%$ with no noise and decreases until $92.4 \%$ with higher noise $(10 \mathrm{~mm})$. 


\begin{tabular}{|c|c|c|c|c|}
\hline $\begin{array}{c}\text { Noise level } \\
(\mathrm{mm})\end{array}$ & $\begin{array}{c}\text { Mean } \\
(\mathrm{mm})\end{array}$ & Standard deviation (mm) & Minimum (mm) & Maximum (mm) \\
\hline 0 & 2.12 & 4.46 & 0.010 & 38.24 \\
\hline 2 & 2.32 & 4.71 & 0.029 & 39.99 \\
\hline 4 & 2.41 & 4.86 & 0.023 & 39.01 \\
\hline 6 & 2.63 & 5.36 & 0.027 & 40.90 \\
\hline 8 & 2.77 & 5.83 & 0.028 & 42.10 \\
\hline 10 & 2.82 & 5.73 & 0.029 & 43.12 \\
\hline
\end{tabular}

Table 1: Statistics of the metrics obtained from 1000 tests and for different level of noise

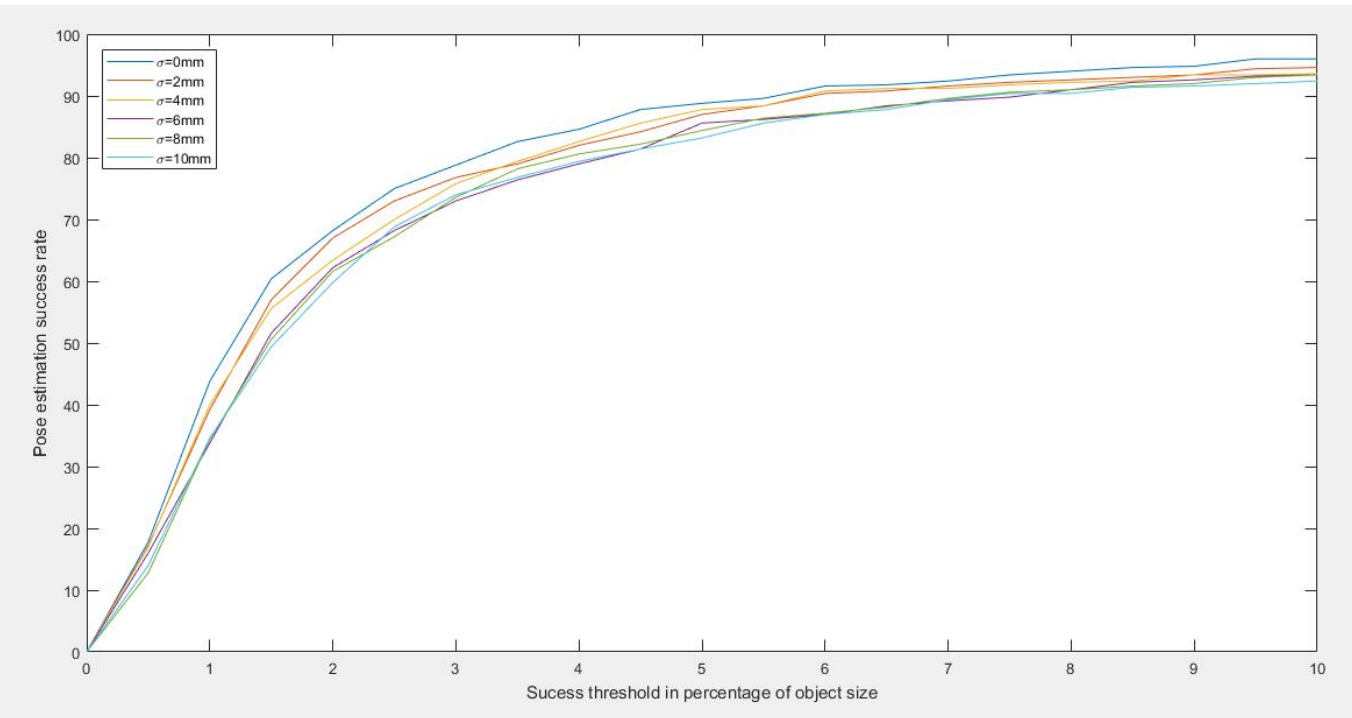

Figure 1: Pose estimation success rate according to $k$

\section{Conclusion}

This preliminary study intended to assess a new approach allowing the markerless localization of surgical instruments for computer assisted orthopaedic surgery. With such approach, low cost depth cameras could be used during surgery and markers could be avoided reducing thus the additional complexities associated to existing surgical navigation systems. The results are very encouraging compared to the state of the art literature. Indeed, [2] and [5] report respectively an higher average registration error of $3.04 \mathrm{~mm}$ and $11.46 \mathrm{~mm}$ on real data. However, the influence of many parameters such as the camera resolution or the type of descriptor has to be further assessed. Similarly, the accuracy and the reliability of our method have to be evaluated in a real clinical environment. 


\section{References}

[1] Manuela Kunz, Balaketheeswaran, Randy Ellis and John Rudan. (2015) The influence of osteophyte depiction in CT for patient specific guided hip resurfacing procedures. International Journal of Computer Assisted Radiology and Surgery 10(6): 717-726.

[2] Sing Chun Lee, Bernhard Fuerst, Keisuke Tateno, Alex Johnson, Javad Fotouhi, Greg Osgood, Federico Tombari and Nassir Navab. (2017) Multi-modal imaging, model-based tracking, and mixed reality visualisation for orthopaedic surgery. Healthcare Technology Letters 4(5): 168-173.

[3] Aitor Aldoma, Markus Vincze, Nico Blodow, David Gossow, Suat Gedikli, Radu Bogdan Rusu and Gary Bradski. (2011). CAD-model recognition and 6DOF pose estimation using 3D cues. Proceedings of the IEEE International Conference on Computer Vision: 585-592.

[4] Yu Xiang, Tanner Schmidt, Venkatraman Narayanan and Dieter Fox. (2017) PoseCNN : A Convolutional Neural Network for 6D Object Pose Estimation in Cluttered Scenes. Proceedings of Robotics: Science and Systems XIV: 19-28.

[5] Jonas Hajek, Mathias Unberath, Javad Foutouhi, Bastian Bier, Sing Chun Lee, Greg Osgood, Andreas Maier, Mehran Armand and Nassir Navab. (2018). Closing the Calibration Loop: An InsideOut-Tracking Paradigm for Augmented Reality in Orthopedic Surgery. Medical Image Computing and Computer Assisted Intervention 2018 (4): 299-3 